\title{
Safety and Efficacy of Balloon Kyphoplasty for Compression Fractures in the Very Elderly
}

\author{
Gurpreet S. Gandhoke ${ }^{1}$, Christopher P. Deibert ${ }^{2}$, Erin Paschel ${ }^{1}$, Peter C. Gerszten ${ }^{1}$ \\ 1. Department of Neurological Surgery, University of Pittsburgh Medical Center 2. University Of \\ Pittsburgh
}

$\square$ Corresponding author: Gurpreet S. Gandhoke, gurpreet.gandhoke@gmail.com Disclosures can be found in Additional Information at the end of the article

\section{Abstract}

Introduction

Balloon kyphoplasty is a well-accepted treatment for symptomatic compression fractures. Concern has been raised regarding unacceptable morbidity and poor outcomes in very elderly patients who undergo the procedure. This study was undertaken to evaluate the safety and efficacy of a series of very elderly patients who underwent balloon kyphoplasty.

\section{Materials Methods}

A retrospective cohort evaluation was performed of a consecutive series of 162 patients over age 80 who underwent treatment for compression fractures between 2006 and 2013 using the balloon kyphoplasty technique. The indication for treatment included pain unresponsive to non-surgical management in all cases.

Results

All procedures were successfully completed under general anesthesia. There were 43 men and 119 women (mean age 88, range 80-99 years). One hundred forty-two fractures were osteoporotic ( 23 had history of trauma), and 20 were pathologic. Fifty patients were treated in an outpatient setting. A total of 253 levels were treated, most common being L1 $(n=38)$ and T12, L2 ( $\mathrm{n}=35$ each). Ninety- one patients had a single level treated, 56 had 2 levels treated, and 15 had 3 levels treated. The range of levels treated spanned from T4 ( $\mathrm{n}=2)$ through L5 $(n=11)$. Back pain improvement was used as the primary outcome measure. The mean VAS score for back pain improved from 9 to 3.5 ( $\mathrm{p}<0.0001)$. There were no cases of surgical site infections, cement extravasation, or new neurologic deficit for the entire cohort. Twenty-nine patients expired due to causes unrelated to the procedure during the follow-up period.

Received 10/15/2013 Review began 10/16/2013 Published 02/23/2014

C) Copyright 2014

Gandhoke et al. This is an open access article distributed under the terms of the Creative Commons Attribution License CC-BY 3.0., which permits unrestricted use, distribution, and reproduction in any medium, provided the original author and source are credited.
Twenty- two (14\%) patients underwent a second procedure for a new compression fracture after the index treatment at a mean interval of 7 weeks; 15 of these $(68 \%)$ were performed at a level adjacent to the index level.

\section{Conclusion}

Balloon kyphoplasty is a safe and highly effective treatment for symptomatic compression fractures, even for very elderly patients. The procedure can be performed in an outpatient setting. Age alone should not be an exclusion criterion for candidate patients. 
Keywords: Balloon kyphoplasty, fracture, osteoporosis, surgery in the elderly, compression fractures

Categories: Neurosurgery

Keywords: balloon kyphoplasty, fracture, osteoporosis, surgery in the elderly, compression fractures

\section{Introduction}

Osteoporosis is an age-related progressive skeletal disease, with consequent loss in bone mass and considerable tendency to fracture [1]. Vertebral compression fractures (VCFs) are the most common type of osteoporotic fractures, resulting in severe back pain, spinal deformity, muscle atrophy, physical decline, prolonged hospitalization, and potential risk of increased mortality $[2,3]$. Osteoporotic vertebral fractures occur in approximately $20 \%$ of people older than age 70 worldwide, with an estimated 1,400,000 new fractures occurring every year [4].

There is currently a substantial body of literature supporting the use of percutaneous balloon kyphoplasty for the treatment of symptomatic compression fractures [5-11]. The procedure is minimally invasive, and it can frequently be performed in an outpatient setting.

Vertebral augmentation refers to vertebroplasty and/or kyphoplasty. Vertebroplasty is a minimally invasive, image-guided procedure that involves the injection of polymethylmethacrylate (PMMA) cement into a fractured vertebral body. Balloon kyphoplasty involves the initial inflation of a balloon tamp that creates a low resistance cavity within the vertebral body, into which cement is subsequently injected [8].

As one might imagine, the majority of patients who undergo balloon kyphoplasty for symptomatic vertebral compression fractures are older individuals. Older individuals not only develop compression fractures due to underlying osteoporosis, but they also develop a relatively higher incidence of fractures due to metastatic disease from underlying malignancy that is more prevalent in the very elderly. The very elderly usually possess a significant number of medical co-morbidities including primary cardiac and pulmonary conditions that elevate their risk for undergoing surgical interventions that requires a general anesthetic. Given the concern regarding a potentially greater morbidity and possibly poor outcomes in very elderly patients who undergo the balloon kyphoplasty procedure, a study was undertaken that specifically evaluated the safety and efficacy of this subgroup.

This study was undertaken to evaluate the safety and efficacy of a series of very elderly patients who underwent balloon kyphoplasty. We hypothesize that balloon kyphoplasty is an effective modality to control pain caused by compression fractures.

\section{Materials And Methods}

\section{Materials}

A retrospective cohort evaluation was performed on a consecutive series of 162 patients over age 80 who underwent treatment for compression fractures between 2006 and 2013 using the balloon kyphoplasty technique. The Kyphon Balloon Kyphoplasty instrumentation was used in all cases (Medtronic, Minneapolis, MN). The indication for treatment included pain thought to be related to the index fracture that was unresponsive to non-surgical management in all cases. This indication was agreed upon by the referring physician and the treating surgeon. There were 43 men and 119 women (mean age 88, range 80-99 years). One hundred forty- two fractures were osteoporotic (23 had history of trauma), and 20 were pathologic. 


\section{Method}

Balloon kyphoplasty is a technique for vertebral compression fractures that involves the introduction of a cannula into the vertebral body followed by the insertion of an inflatable balloon. The balloon is inflated within the vertebral body. When the balloon is inflated, it creates a space within the center of the vertebral body that also elevated the end plate fractures. PMMA is then injected under low pressure into the cavity left by the balloon. It is this fixation of the fracture that is believed to allow for pain improvement. In addition, the inflation of the balloon itself during the procedure can also lead to some increase in the vertebral body height and therefore correction of the abnormal configuration of the vertebral body that existed as a result of the fracture. After the balloon is removed, there is an empty space within the vertebral body that allows for the low-pressure injection of the PMMA into the cavity created by the balloon. Injection under low pressure in kyphoplasty has the advantage of decreasing the rate of leakage of cement either into the spinal canal or into the draining veins of the vertebral body.

\section{Technical success}

Technical success was determined by the ability of the cement to increase the height of the compressed vertebral body. Success was also determined by the ability of making the cement reach at the most compressed portion of the vertebral body without extravasation out of the confines of the vertebral body or into the paravertebral veins. (As can be seen in Figures 1-4.)

\section{Results}

All procedures were successfully completed under general anesthesia. The procedures were performed by the senior author PCG who has been performing this procedure starting from 2001 for the last 12 years. Fifty-two patients were treated in an outpatient setting. No procedure had to be aborted due to medical issues at the time of surgery. Co-morbidities included history of diabetes $(n=21)$, hypertension $(n=109)$, prior pulmonary embolism $(n=11)$, and coronary artery disease $(n=61)$. A total of 253 levels were treated, most common being L1 $(n=38)$ and T12 and L2 ( $n=35$ each). Ninety-one patients had a single level treated, 56 had 2 levels treated, and 15 had 3 levels treated. Range of levels treated spanned from T4 (n=2) through L5 (n=11).

Improvement in back pain was the primary outcome measure employed. The mean VAS score for back pain improved from 9 to 3.5 for the entire cohort $(\mathrm{p}<0.0001)$. The VAS scores were determined at the last follow up and were done in clinic by the surgeon himself and the neurosurgery resident evaluating the patient in follow up clinic. There were no cases of infections, cement extravasation, or new neurologic deficit in any of the cases. Twenty-nine patients expired due to causes unrelated to the procedure during the follow-up period. There were no deaths attributed to the surgical procedure or anesthetic. Twenty-two (14\%) patients underwent a second procedure at a mean interval of 7 weeks; 15 of these (68\%) were performed at a level adjacent to the index level. 


\section{Cureus}

\begin{tabular}{|c|c|c|c|}
\hline Demographics & Co-Morbidities & Etiology & Levels Treated \\
\hline Male: 43 & Hypertension: $n=109(67 \%)$ & Osteoporosis: n=142 (88\%) & Total levels: $n=253$ \\
\hline Female: 119 & Diabetes: n=21 (13\%) & Pathologic: $n=20$ & Single level: $n=91$ \\
\hline \multirow[t]{2}{*}{ Mean age: 88 (range 80-99) } & CAD: $n=61(38 \%)$ & & Two levels: $n=56$ \\
\hline & PE: $\mathrm{n}=11(7 \%)$ & & Three levels: $n=15$ \\
\hline
\end{tabular}

\section{TABLE 1: Breakdown of results}

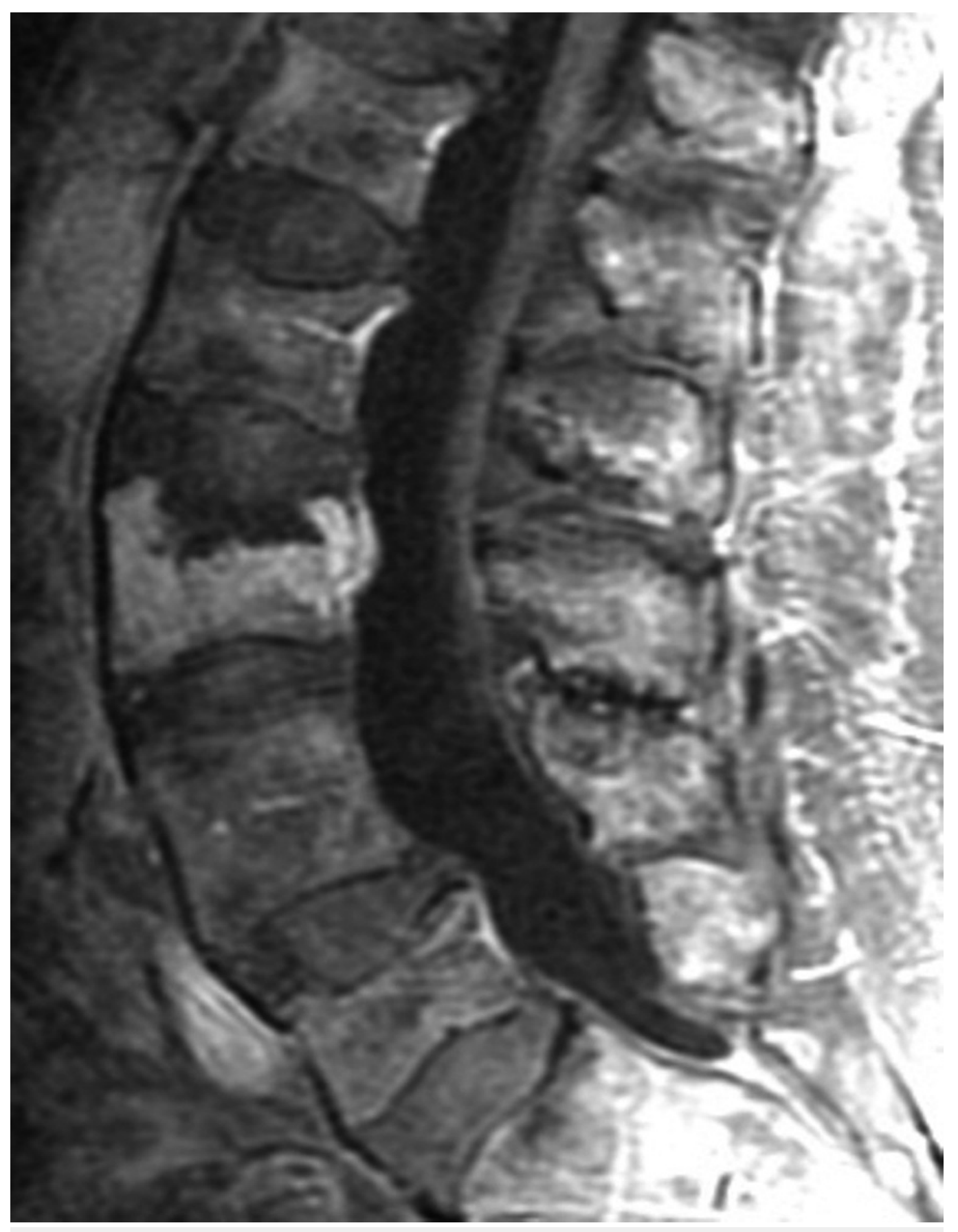




\section{Cureus}

FIGURE 1: Case example of an 85 year old woman with a symptomatic L3 compression fracture secondary to osteoporosis. MR imaging of the fracture.

Case example of an 85 year old woman with a symptomatic L3 compression fracture secondary to osteoporosis. MR imaging of the fracture.

FIGURE 2: Intraoperative lateral flouroscopic image depicting the inflated balloons within the L3 vertebral body fracture.

Intraoperative lateral flouroscopic image depicting the inflated balloons within the L3 vertebral body fracture. Notice the reduction of the superior endplate fracture. 


\section{Cureus}

FIGURE 3: Lateral radiograph demonstrating the PMMA filling the L3 vertebral body, especially underneath the superior endplate fracture.

Lateral radiograph demonstrating the PMMA filling the L3 vertebral body, especially underneath the superior endplate fracture. 


\section{Cureus}

FIGURE 4: AP radiograph demonstrating the PMMA filling both sides of the L3 vertebral body in the location of the previously inflated balloons.

AP radiograph demonstrating the PMMA filling both sides of the L3 vertebral body in the location of the previously inflated balloons. The patient was discharged to home on the day of the procedure with complete back pain resolution.

\section{Discussion}

Osteoporosis affects greater than $55 \%$ of women older than age 55 and is characterized by decreased bone mineral density resulting in increased bone fragility and a higher susceptibility to fracture [11]. In the United States, osteoporosis leads to over 700,000 vertebral compression fractures per year. Treatment of osteoporosis and associated fractures incurs a cost exceeding $\$ 17$ billion each year $[12,13]$. Most osteoporotic vertebral compression fractures are asymptomatic or result in minimal pain [8]. Between 10 and 20\% result in hospitalization.

Vertebral fractures in cancer patients may be secondary to osteoporosis, primary neoplasm, 
metastasis, osteopenic effects of drug therapy, or effects of radiation therapy. Reduced bone mineral density is found in nearly half of all patients diagnosed with cancer and osteoporosis is more common when compared to the general population [14]. Fractures in patients with cancer cause significant exacerbation of local pain and can be disabling. Vertebral augmentation has been determined to be a very safe and effective treatment option for cancer patients presenting with a symptomatic pathological fracture [15-17]. The incidence of cancer and of spine metastases increases with age and is prevalent among the very elderly.

The initial treatment of VCF includes activity modification and analgesic medications [11]. Many patients sustain serious cardiovascular, musculoskeletal, metabolic, and immune complications related to immobility and bed rest [18]. Elderly patients typically affected by VCF often cannot tolerate the complications of non-operative care. These elderly patients can be admitted to a hospital for treatment, discharged, and readmitted to treat complications with medical resources used at each stage [11,19]. The result can be a downward spiral of complications, functional decline, and a higher risk of death as a result of the VCF for this fragile subgroup of patients [3,20-22]. It is for this reason that our facility has favored an earlier threshold to treat these symptomatic patients with balloon kyphoplasty. Vertebral augmentation is typically considered for patients presenting with a symptomatic vertebral fracture that results in severe disabling back pain, marked reduction in mobility, and quality of life [8].

Treatment of VCF in the very elderly that accelerates the return of patient function can potentially reduce both the medial risks as well as the economic burden of the disease [11]. It is for this reason that there has been such an interest in the adoption and evaluation of percutaneous vertebral augmentation for the management of VCF. In the last five years alone, over 300 articles have been published annually on vertebral augmentation [8]. Nevertheless, there remains much debate regarding the safety as well as efficacy to achieve analgesia, reduce disability, and improve quality of life in patients with a vertebral fracture.

The current study was undertaken to specifically evaluate the use of the balloon kyphoplasty procedure in a consecutive longitudinal cohort of patients treated at a single institution by single surgeon over seven years. With the increasing scrutiny of major medical outcomes with spinal augmentation versus conservative therapy [23], an analysis of the peri-operative morbidity of patients older than 80 who undergo balloon kyphoplasty would be of value to this debate. In the current series, the procedure was successfully performed under a general anesthetic without peri-operative morbidity. The procedure could be successfully performed on an outpatient basis for a significant percentage of the cohort. Many of our patients were already hospitalized for pain due to their fracture and therefore could not be treated on an outpatient basis. Otherwise, the number of outpatient procedures would have been much greater for the cohort.

The overall percentage of major medical co-morbidities including diabetes mellitus, hypertension, coronary artery disease, and a history of pulmonary embolism requiring anticoagulation for group of patients was substantial, and it reflects the co-morbidities that afflict patients over the age of 80. Regarding the safety of general anesthesia in this cohort, it should be pointed out that these cases were performed at a tertiary medical center with dedicated neuro-anesthesiologists who are highly experienced in caring for such elderly patients with significant medical co-morbidities. Nevertheless, this study reveals that these short procedures which are performed with minimal blood loss are well tolerated by these very elderly patients, even in the setting of major medical co-morbidities or underlying cancer.

This study documented a statistically significant substantial and sustained improvement in spinal pain after the balloon kyphoplasty procedure based upon a visual analog scoring system 
similar to that seen in previously published meta-analysis studies of vertebral augmentation $[1,7,10]$. These clinical outcomes are no different than those reported for lower age groups with similar pathology. Furthermore, there were no cases of surgical site infections, cement extravasation, or new neurologic deficits that resulted from the procedure. Once again, there is no higher incidence of procedural complications in the very elderly compared to lower age groups undergoing the same vertebral augmentation procedure.

\section{Conclusions}

Balloon kyphoplasty is a safe and highly effective treatment for symptomatic compression fractures, even for very elderly patients. The procedure can be performed successfully in an outpatient setting. Age alone should not be an exclusion criterion for candidate patients.

\section{Additional Information \\ Disclosures}

Human subjects: All authors have confirmed that this study did not involve human participants or tissue. Animal subjects: All authors have confirmed that this study did not involve animal subjects or tissue. Conflicts of interest: In compliance with the ICMJE uniform disclosure form, all authors declare the following: Payment/services info: All authors have declared that no financial support was received from any organization for the submitted work. Financial relationships: All authors have declared that they have no financial relationships at present or within the previous three years with any organizations that might have an interest in the submitted work. Other relationships: All authors have declared that there are no other relationships or activities that could appear to have influenced the submitted work.

\section{References}

1. Shi MM, Cai XZ, Lin T, Wang W, Yan SG: Is there really no benefit of vertebroplasty for osteoporotic vertebral fractures? A meta-analysis. Clinical orthopaedics and related research. 2012, 2012:2785-279.

2. Bliuc D, Nguyen ND, Milch VE, Nguyen TV, Eisman JA, Center JR: Mortality risk associated with low-trauma osteoporotic fracture and subsequent fracture in men and women. JAMA. 2009, 301:513-521.

3. Kado DM, Browner WS, Palermo L, Nevitt MC, Genant HK, Cummings SR: Vertebral fractures and mortality in older women: a prospective study. Study of Osteoporotic Fractures Research Group. Archives of internal medicine. Archives of internal medicine. 1999, 159:1215-1220.

4. Johnell O, Kanis JA: An estimate of the worldwide prevalence and disability associated with osteoporotic fractures. Osteoporosis international. 2006, 17:1726-1733.

5. Borgstrom F, Olafsson G, Strom O, et al.: The impact of different health dimensions on overall quality of life related to kyphoplasty and non-surgical management. Osteoporosis international. 2013, 24:1991-1999.

6. Borgstrom F, Lekander I, Ivergard M, et al.: The International Costs and Utilities Related to Osteoporotic Fractures Study (ICUROS)--quality of life during the first 4 months after fracture. Osteoporosis international. 2013, 24:811-823.

7. Albers SL, Latchaw RE: The effects of randomized controlled trials on vertebroplasty and kyphoplasty: a square PEG in a round hole. Pain physician. 2013, 16:E331-348.

8. Chandra RV, Yoo AJ, Hirsch JA: Vertebral augmentation: update on safety, efficacy, cost effectiveness and increased survival. Pain physician. 2013, 16:309-320.

9. Saxena AP, Hirsch AE, Yoo AJ, Gilligan CJ, Hirsch JA: The use of advanced imaging and representation of workers compensation in vertebral augmentation: a single-center comparison with the INVEST Trial. Pain physician. 2013, 16:E391-396.

10. Anderson PA, Froyshteter AB, Tontz WL, Jr: Meta-analysis of vertebral augmentation compared with conservative treatment for osteoporotic spinal fractures. Journal of bone and mineral research. 2013, 28:372-382.

11. Zampini JM, White AP, McGuire KJ: Comparison of 5766 vertebral compression fractures 


\section{Cureus}

treated with or without kyphoplasty. Clinical orthopaedics and related research. 2010, 468:1773-1780.

12. Melton LJ 3rd.: Epidemiology of spinal osteoporosis. Spine. 1997, 22:2S-11S.

13. Truumees E: Osteoporosis. Spine. 2001, 26:930-932.

14. Reuss-Borst M, Hartmann U, Scheede C, Weiss J: Prevalence of osteoporosis among cancer patients in Germany: prospective data from an oncological rehabilitation clinic. Osteoporosis international. 2012, 23:1437-1444. 